\title{
Analysis of Residual Stress in Stress Harps of Grey Iron by Experiment and Simulation
}

\author{
Pål Schmidt ${ }^{1, a}$, Ru Lin Peng ${ }^{2, b^{*}}$, Vadim Davydove,c, Mattias Lundberg' ${ }^{2, d}$ \\ Maqsood Ahmad $^{1, \mathrm{e}}$, Taina Vuoristo ${ }^{4, \mathrm{f}}$, Daniel Backström ${ }^{4, g}$ \\ Sten Johansson ${ }^{2, h}$ \\ 'Volvo Group Trucks Technology, Göteborg, Sweden \\ ${ }^{2}$ Department of Management and Engineering, Linköping University, Linköping, Sweden \\ ${ }^{3}$ Paul Scherrer Institute, Spallation Neutron Source SINQ, Villigen PSI, Switzerland \\ ${ }^{4}$ UTMT, Scania CV AB, Södertälje, Sweden

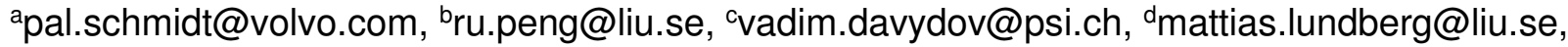 \\ emaqsood.ahmad@volvo.com, ${ }^{\mathrm{f}}$ taina.vuoristo@scania.com, 9Daniel.Backstrom@scania.com, \\ hsten.johansson@liu.se
}

Keywords: Residual stress, Cast iron, FE simulation, Neutron diffraction, Hole drilling

\begin{abstract}
Stress harps with bars of different size were used to study residual stresses due to different cooling rate during casting of a grey iron. Finite element (FE) simulations were performed to predict residual stresses from the casting process and the effect of a stress relieving heat treatment. Intended for validating the simulations, neutron diffraction (ND) and hole drilling methods were used to measure the residual stress distribution through the thickness and in a thin surface layer, respectively. Good agreement between the FE simulations and ND measurements is observed for the annealed harp and the normal and transverse directions of the as cast harp. Discrepancy occurs in the axial direction and especially in the side bars of the as cast harp for which the simulation shows much higher compressive residual stresses. The observed difference between the different techniques was discussed with respect to the characteristics of the different methods.
\end{abstract}

\section{Introduction}

Cast irons are widely used for casting components in heavy duty diesel engines. During the casting process differential cooling may occur in different parts of a casting. Cooling induced thermal contractions and deformation therefore vary within the casting and below a so called stress-free temperature internal stresses start to build up. At the end of the cooling process, compressive residual stresses are expected to be retained in those regions of faster cooling but tensile stresses in those regions of slower cooling. Thermal residual stresses in cast irons are undesirable as the tensile residual stresses can cause cracking of the castings. They can also reduce the fatigue strength by adding on the applied load or assist stress corrosion cracking.

Numerical simulation of thermal and mechanical processes is an attractive tool which can generate detailed residual stress maps and is gaining increased importance for residual stress analysis in castings. The accuracy of such simulation depends among others on how close the mechanical and thermal behavior of the casting system can be modeled and the availability of material data over a large temperature range which can be difficult to obtain. Experimental validations of the implemented models are therefore important. Among the established experimental evaluation methods, X-ray diffraction (XRD), neutron diffraction (ND) and hole-drilling (HD) techniques are commonly used. Residual stress measurements by both XRD and HD methods are often confined to a thin surface layer of less than $1 \mathrm{~mm}$ while ND, with the penetration power of neutrons in cast irons, can probe residual stresses at a larger depth. However, measurements in components of complex geometry are not trivial since the neutron beam paths can easily reach beyond the penetration limit of neutrons. 
Because of their simple geometry, stress harps/lattices containing interconnected bars of different size have been used to study casting/thermal residual stresses in different metallic materials and used to validate numerical simulations. Very good agreement between simulation and neutron measurements was reported for a $10 \mathrm{~mm}$ thick stress lattice of ductile iron [1]. In the current work, we used relatively thick, $25 \mathrm{~mm}$, stress harps (Fig.1) to study thermal residual stresses from differential cooling of a cast grey iron and the influence of a stress relieving heat treatment. Finite element (FE) simulation was carried out in an as cast and an annealed stress harp and compared with ND measurements and HD measurement in the near surface region of the centre bar.

The nominal chemical composition and tensile strength of the cast iron GJL-300 are given in Table 1. The microstructure consists of flake graphite in a matrix of lamellar pearlite. The two harps were cast in chemically bonded sand moulds (furane) and knocked out at $750{ }^{\circ} \mathrm{C}$ (ID 10) and room temperature (ID 11). The annealing treatment of harp No. 10 was done at $600{ }^{\circ} \mathrm{C}$ with 3 hours holding time, followed by controlled cooling down below $300{ }^{\circ} \mathrm{C}$ and then natural cooling in air.

Table 1. Nominal chemical composition (wt\%) and mechanical properties of GJL-300.

\begin{tabular}{|l|l|l|l|l|l|l|l|l|l|}
\hline $\mathrm{C}$ & $\mathrm{Si}$ & $\mathrm{Mn}$ & $\mathrm{P}$ & $\mathrm{S}$ & $\mathrm{Cr}$ & $\mathrm{Mo}$ & $\mathrm{Cu}$ & $\mathrm{Fe}$ & UTS (MPa) \\
\hline 3,2 & 1,8 & 0,6 & 0,05 & 0,07 & 0,15 & 0,2 & 0,9 & bal & 290 \\
\hline
\end{tabular}

\section{Simulation}

The simulation was carried out with Magmasoft v5.1 using the iron module. The total number of cells used to model a half stress harp was 500000 (including mould). The pouring temperature was set to $1400{ }^{\circ} \mathrm{C}$ and heat transfer coefficient to $500 \mathrm{~W} / \mathrm{m}^{2}, \mathrm{~K}$. Mould filling was not included in the simulation. Since the heat treatment module was not available creep mode was activated in order to simulate the influence of annealing. The material properties were taken from Magmasoft's database.

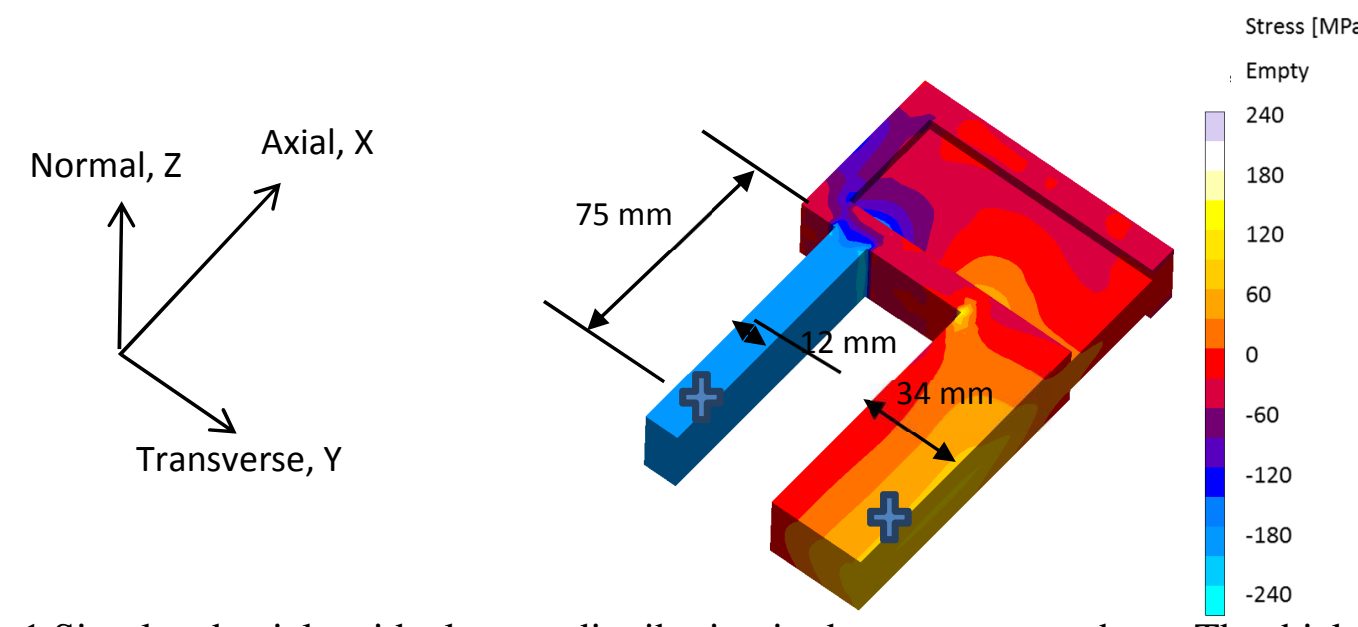

Fig. 1 Simulated axial residual stress distribution in the as cast stress harp. The thickness of the stress harp is $25 \mathrm{~mm}$ and the total length is $295 \mathrm{~mm}$.

The calculated distribution of the axial residual stress is shown in Fig. 1 for a quarter of the as cast stress harp. Stress components at selected positions of the as-cast and annealed harps are compared with the ND measurements in Figs. 3 and 4 in Section 3.2. As expected, compressive residual stresses develop in the side bar which cools faster because of the small cross-section and tensile stresses appear in the centre bar which having a large cross-section cools slower. The tensile residual stresses in the centre bar vary largely from high tension in the mid-width to low tension towards the edge. Such a distribution is due to a large width to thickness ratio giving different cooling rates between the edge and the middle. 


\section{Residual stress measurement}

\subsection{Hole drilling measurements}

Axial residual stress distributions at the centreline of the middle bar were measured. The set-up consists of a rosette strain gauge with 3 strain gauges at $45^{\circ}$ to each other and the centre one is always oriented parallel to the bar of the harp which happens also to be the maximum principal stress direction (Fig. 2). A hole was drilled with a $\Phi 1.8 \mathrm{~mm}$ drill in the centre of the rosette down to a depth of $1 \mathrm{~mm}$ and the measured strain changes were re-calculated into a residual stress-depth profile by the Kockelmann method [2]. The Young's modulus was set to $110 \mathrm{GPa}$ and Poisson's ratio to 0.3 . As can be seen, a more drastic residual stress variation than the simulation with high values near surface but very low value in the subsurface is obtained for the as cast harp. On the other hand, very good agreement (the circle from the simulation) is found for the annealed harp.

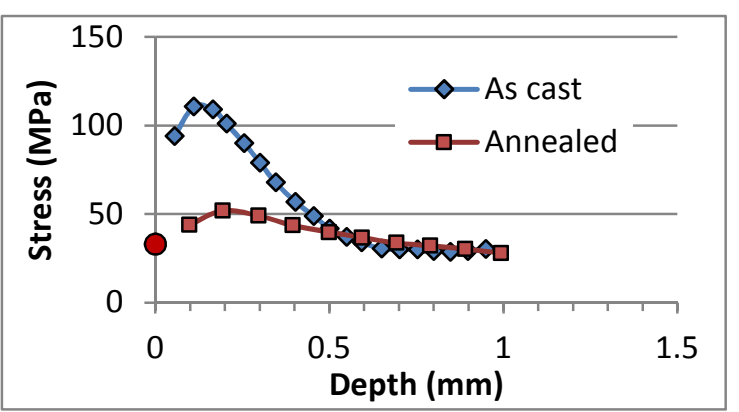

\subsection{Neutron diffraction measurements}

The neutron diffraction experiment was performed on the stress diffractometer POLDI, a time-offlight instrument, at PSI in Villigen, Switzerland. Lattice strains along the principle directions of the harp were measured at three depths, $2.5,12.5$ and $22.5 \mathrm{~mm}$ beneath the marked surface, indicated in Fig.1. Because of the large thickness, a large gauge volume, $3.8 \times 3.8 \times 10 \mathrm{~mm}^{3}$, defined by the incident and diffracted beam slits, was used. The height of the gauge volume, $10 \mathrm{~mm}$, is oriented vertically in the diffractometer. The stress free lattice parameter was based on measurements of two small bars with $10 \times 10 \mathrm{~mm}^{2}$ cross-section cut by EDM from a grey cast iron and adjusted slightly to minimize the residual stresses in the normal direction. The obtained neutron spectra were fitted using PSI's in-house software to determine the position of a number of ferrite peaks but the lattice strains were derived from the ferrite 211 peaks having the best quality. Residual stresses were then calculated from the lattice strains using the Hooke's law and the Young's modulus and Poisson's ratio of Fe-211 which are $225 \mathrm{GPa}$ and 0.285 [3].

Similar to the simulation, compressive axial residual stresses are found in the side bar and tensile in the center bar of the as cast stress harp (Fig. 3a). Variation of the residual stresses with depth, being higher near the surfaces, is also observed. Residual stresses in the normal and transverse directions, shown in Figs. $3 \mathrm{~b}$ and c, are in general low but with an exception at the depth of 22.5 $\mathrm{mm}$. The high transverse stress at this point is related to a large peak shift measured in the transverse direction. As the diffraction peak didn't show any abnormity and the peak intensity was comparable to that at the $2.5 \mathrm{~mm}$ depth, this data point is treated as an outlier.

Fig. 4 shows low residual stresses in the annealed harp. Considering the uncertainty range, it can be derived that the heat treatment affected mostly the axial residual stresses near the surface region. 


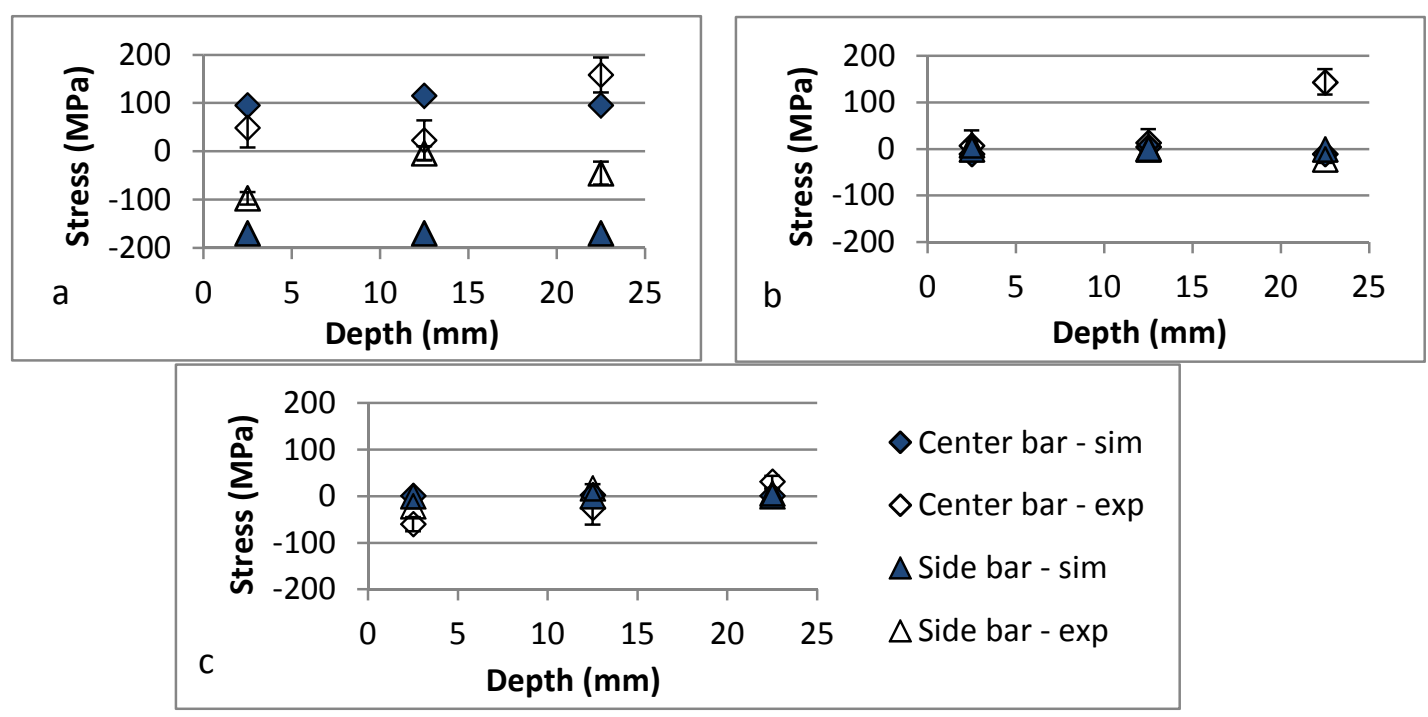

Fig. 3 Simulation and neutron diffraction measurements for the as cast stress harp in (a) axial, (b) transverse and (c) normal directions. The point at $22.5 \mathrm{~mm}$ depth is excluded from the analyses.

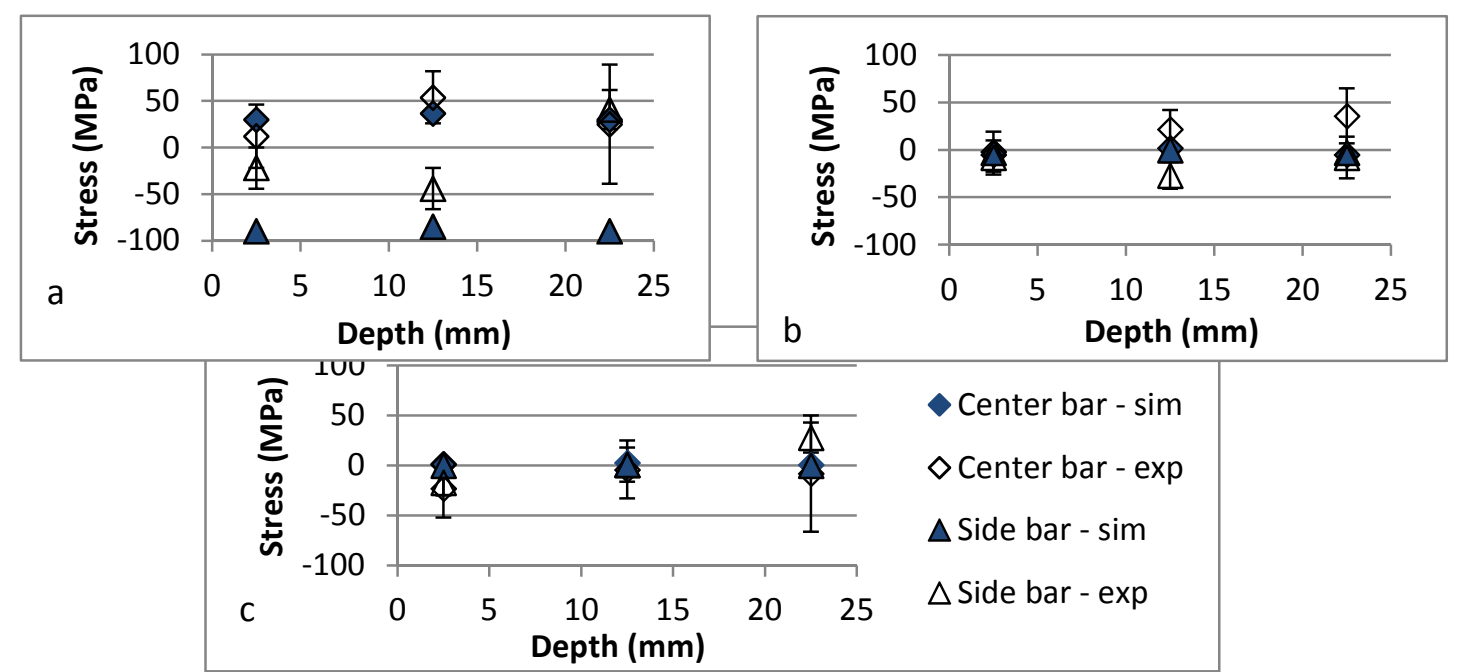

Fig. 4 Simulation and neutron diffraction measurements for the annealed stress harp in (a) axial, (b) transverse and (c) normal direction.

\section{Discussions}

For the annealed harp (Fig. 4) and the normal and transverse directions of the as cast harp (Figs. 3(b) and (c) for which residual stresses are low, the simulations agree reasonably well with the ND measurements within the uncertain range of measurement. However, discrepancy occurs in the axial direction and especially in the side bars of the as cast harp the simulation shows much higher compressive residual stresses. The inherent difference between the two methods is considered to contribute partly to the observed difference as to be further discussed below.

Grey cast iron has a complex microstructure of flake graphite, lamellar ferrite and cementite. Because of their different thermal expansion coefficients and mechanical properties, interactions during cooling can result in microstresses that vary among the phases, as have been shown in different publications on multiphase materials including pearlite, see for example [4]. The FE simulation, upon assuming a homogeneous material, uses macroscopic material properties when modeling the thermal and mechanical process during the solidification and the following cooling. The residual stresses generated are therefore macroscopic and can be considered as some kind of averaged stresses over all the phases. Elastic equilibrium between the side and centre bars is maintained by the macrostresses in the axial direction. On the other hand, the ND technique 
measures residual stresses only in the ferritic phase, which is the sum of the macroscopic stresses and microstresses mentioned above. Relationships between the different kinds of residual stress can be expressed as [3]:

$$
\begin{gathered}
\sigma_{M}=\sum_{i} \phi^{i} \sigma_{p}^{i} \\
\sigma_{p}^{i}=\sigma_{M}+\sigma_{m}^{i}
\end{gathered}
$$

where $\sigma_{\mathrm{M}}$ is the macrostress, $\sigma_{\mathrm{m}}^{\mathrm{i}}$ the microstress for a particular phase $\mathrm{i}$ with volume fraction $\phi^{i}$ and $\sigma_{p}^{i}$ the phase-specific stress measured from a diffraction measurement. As can be seen, a true comparison with the FE results would also require evaluation of residual stresses in the graphite and cementite to derive the macroscopic residual stresses. Due to weak diffraction peaks, residual stress measurements in such phases are difficult. Lacking of experimental results, a qualitative analysis is proposed instead to explain the implications of phase-dependent microstresses for residual stresses due to the differential cooling.

We first consider residual stresses originating from thermal mismatch between the phases. Without any external stress, macrostresses in Eq. (1) are zero. The phase-specific thermal stresses in Eq. (2) are then the same as the microstresses. Because of the low stiffness of graphite, $8.5 \mathrm{GPa}$ against $209 \mathrm{GPa}$ for iron [5] and $200 \mathrm{GPa}$ for the cementite [6], thermal residual stresses related to the graphite are likely very low and negligible. In the matrix, residual microstresses are tensile in the ferrite and compressive in the cementite having a high and a lower thermal expansion coefficient, respectively [7]. Secondly, the macrostresses related to differential cooling rate between the side and centre bars can be treated as "external load". The influence of the microstresses can then be analyzed by making analogy with analysis of load partitioning in a steel plate with surface compressive residual stress and subsurface tensile residual stress [8] in which the surface started to carry the applied load only after its compressive residual stress was canceled by the applied tensile stress. In the side bar of the stress harp, the ferrite is subjected to the compressive "load" only after its residual microstress is canceled. According to Eq.(2), this will shift the phase-specific stress towards a lower tension, and thus partly causes the observed larger difference between the FE and ND results in the side bar. For the centre bar, because of its lower cooling rate, the development of microstresses lags behind the side bars, leading to a lower degree of inhomogeneous load partitioning. Furthermore, the ferrite is softer at a higher temperature and in regions around graphite tips which stress concentration is significant the ferrite may experience plasticity, which causes transfer of part of the tensile "load" back to the cementite [8]. Therefore a smaller difference between the simulation and ND measurements is observed in the centre bar.

Although the difference in thermal coefficient is relatively large between cementite and ferrite, due to the high volume ratio between ferrite and cementite, residual microstresses in the ferrite may not be large. Reported thermal microstresses in ferrite of pearlitic steels vary from almost zero to 50 $\mathrm{MPa}$ in annealing condition after cold drawing [9,10]. This means that the influence of microstresses alone may not explain the observed discrepancy. Giving that the hole drilling measures a low subsurface residual stress already at $0.5 \mathrm{~mm}$ depth in the centre bar, it is likely that the simulation overestimate slightly the magnitude of casting residual stresses in the stress harp.

\section{Conclusions}

Residual stresses were analysed in an as cast and an annealed stress harp by finite element modelling and the results were compared with neutron diffraction and hole drilling measurements.

For the as cast harp, good agreement was found between the simulation and neutron diffraction measurements in the normal and transverse directions with low residual stresses. Discrepancy occurs in the axial direction and especially in the side bars for which the simulation shows much higher compressive residual stresses. All the three methods give low residual stresses in the annealed harp. 
The current work shows that validation of the numerical models for grey cast irons by diffraction method is not straightforward. Residual microstresses due to thermal and mechanical mismatch at microstructural level and the anisotropic behaviour related to the flake graphite need to be considered.

\section{Acknowledgement}

Financial supports from VINNOVA - Sweden's Innovation Agency through the program FFI Strategic Vehicle Research and Innovation are greatly appreciated. Faculty Grant AFM (Faculty Grant SFO-MAT-LiU\#2009-00971) and Agora Materiae Graduate School at Linköping University are also acknowledged for their support to the research work.

\section{References}

[1] E.M. Johnson, T.R. Watkins, J.E. Schmidlin, S.A. Dutler, A benchmark study on casting residual stress, Metallurgical and Materials Transactions A. 43 (2012) 1487-96.

[2] T. Schwarz, H. Kockelmann, The hole-drilling method - the best technique for the experimental determination of residual stresses in many fields of application, MTB 29. 2(1993) 33-38.

[3] I.C. Noyan, J.B. Cohen, Residual Stress Measurement by Diffraction and Interpretation, (1987).

[4] J.M. Atienza, J. Ruiz-Hervias, M. Martinez-Perez, F.J. Mompean, M. Garcia-Hernandez, M. Elices, Residual stresses in cold drawn pearlitic rods, Scr. Mater. 52 (2005) 1223-8.

[5] H. Era, K. Kishitake, K. Nagai, Z.Z. Zhang, Elastic modulus and continuous yielding behaviour of ferritic spheroidal graphite cast iron, Materials Science and Technology. 8 (1992) 257-61.

[6] A.P. Miodownik, Young's modulus for carbides of $3 \mathrm{~d}$ elements (with particular reference to Fe3C), Materials Science and Technology. 10 (1994) 190-2.

[7] F. Laszlo, H. Nolle, On some physical properties of cementite, J. Mech. Phys. Solids. 7 (1959) 193-"194,IN1-IN2,195-198,IN3-IN4,199-208".

[8] R. Lin Peng, J. Gibmeier, G.C. Chai, S. Johansson, Load partitioning in a duplex stainless steel with surface strength gradient and residual stresses, Advanced in X-ray Analysis. 52 (2009) 773-780.

[9] R.A. Winholtz, J.B. Cohen, Changes in the macrostresses and microstresses in steel with fatigue, Materials Science \& Engineering A. A154 (1992) 155-63.

[10] K. Van Acker, J. Root, P. Van Houtte, E. Aernoudt, Neutron diffraction measurement of the residual stress in the cementite and ferrite phases of cold-drawn steel wires, Acta Materialia. 44 (1996) 4039-49. 